J. Phys. IV France 131 (2005) 221-222

(C) EDP Sciences, Les Ulis

DOI: $10.1051 /$ jp4:2005131054

\title{
Electrical transport through constrictions in the charge-density wave conductor $\mathrm{NbSe}_{3}$
}

\author{
K. O'Neill ${ }^{1}$, E. Slot ${ }^{1}$, R. Thorne ${ }^{2}$ and H. van der Zant ${ }^{1}$ \\ ${ }^{1}$ Kavli Institute of Nanoscience Delft, Delft University of Technology, Lorentzweg 1, \\ 2628CJ Delft, The Netherlands \\ ${ }^{2}$ Laboratory of Atomic and Solid State Physics, Clark Hall, Cornell University, Ithaca, \\ New York 14853-2501
}

\begin{abstract}
We have investigated the electrical transport properties of insulating and metallic constrictions of dimensions $100 \mathrm{~nm}-10 \mu \mathrm{m}$ in the charge-density wave (CDW) conductor $\mathrm{NbSe}_{3}$. The constrictions are made in a variety of ways: focused ion beam, reactive ion etching through a resist mask, and in a mechanicallycontrolled break junction configuration. We find that the behaviour of the junctions is independent of the fabrication method, and, depending on the size of the constriction, that the low-temperature behaviour of the constrictions is metallic or insulating. At $4.2 \mathrm{~K}$ we observe peaks in the differential conductance near $100 \mathrm{mV}$ and $200 \mathrm{mV}$, which vanish as the temperature is increased above the Peierls temperatures $59 \mathrm{~K}$ and $145 \mathrm{~K}$ respectively. The data is consistent with the interpretation that there are two different regimes, depending on the size of the constriction, demonstrating either tunnelling behaviour, due to CDW-insulator-CDW junctions, or metallic behaviour, due to two normal metal-CDW junctions in series.
\end{abstract}

\section{INTRODUCTION}

Recent advances in fabrication techniques allow the study of mesoscopic aspects of charge-density wave (CDW) transport [1,2]. Since tunnelling through fully-gapped CDW conductors, like blue bronze, suffer from band-bending effects at the interface akin to semiconductor-insulator-metal junctions, work is limited to $\mathrm{NbSe}_{3}$, which remains metallic down to $4.2 \mathrm{~K}$. Previous in-chain tunnelling studies and mechanical point contact experiments have either not extended to sufficiently high bias ranges to observe energy-gap related features or have been cited as having poor stability [1, 3]. We present here transport results of constrictions between $\sim 100 \mathrm{~nm}$ and $10 \mu \mathrm{m}$ in $\mathrm{NbSe}_{3}$ formed using three different approaches: Focused Ion Beam (FIB) fabrication, Resist Mask and subsequent reactive ion etched (RM) fabrication, and Mechanically Controlled Break Junction (MCBJ) samples. More details on these approaches may be found in [4]. Broadly speaking, we observe two kinds of low-temperature behaviour, depending on the size of the junction: in sufficiently small junctions, and broken MCBJ samples, the differential conductance $\mathrm{dI} / \mathrm{dV}$ is minimum at zero bias; in larger junctions, and re-contacted MCBJ samples, $\mathrm{dI} / \mathrm{dV}$ is maximum at zero bias. Common to all junctions is the observation of peaks in $\mathrm{dI} / \mathrm{dV}$ at biases symmetric around $\sim 100 \mathrm{mV}$ and $\sim 200 \mathrm{mV}$.

\section{RESULTS AND DISCUSSION}

Figure 1 shows data collected from FIB, MCBJ and RM samples of increasing size. Fig 1(a) shows results for a FIB constriction of size $<100 \mathrm{~nm}$ : $\mathrm{dI} / \mathrm{dV}$ is suppressed around zero bias and shows peaks at $\pm 105 \mathrm{mV}$ and $\pm 195 \mathrm{mV}$. This behaviour is reproduced in a MCBJ after breaking, also shown in Fig. 1(a). The zero bias resistance versus temperature, shown in Fig. 1(b), decreases monotonically and bears little resemblance to the bulk behaviour of $\mathrm{NbSe}_{3}$, which is metallic down to $4.2 \mathrm{~K}$. The peak positions, Fig. 1(c), also decrease monotonically with temperature, indicating a strict association between each $\mathrm{dI} / \mathrm{dV}$ peak and the lower and upper CDW transitions in bulk $\mathrm{NbSe}_{3}$ at $59 \mathrm{~K}$ and $145 \mathrm{~K}$. Figure $1(\mathrm{~d})$ shows 

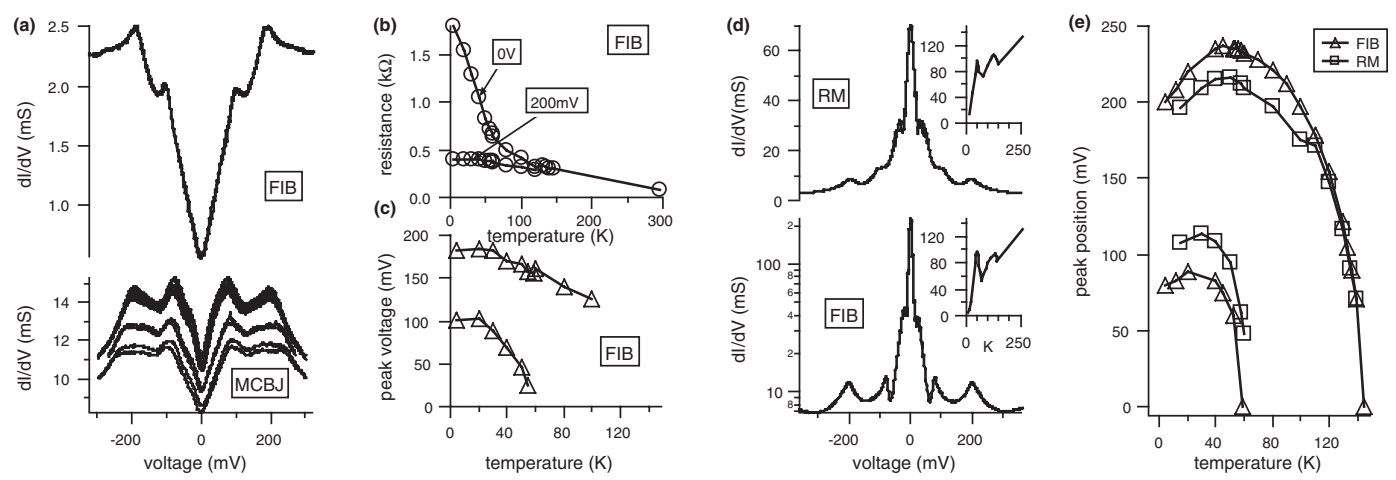

Figure 1. (a) $\sim 100 \mathrm{~nm}$ FIB constrictions and broken MCBJ sample $\mathrm{dI} / \mathrm{dV}$ vs bias voltage, $4.2 \mathrm{~K}$; (b) $\mathrm{R}(\mathrm{T}$ ) at $0 \mathrm{~V}$ and $200 \mathrm{mV}$ of FIB sample in (a); (c) Temperature variation of peak voltages of FIB sample in (a); (d) $\sim 1 \mu \mathrm{m} \mathrm{RM}$ and $8 \mu \mathrm{m}$ FIB sample $\mathrm{dI} / \mathrm{dV}$ vs bias voltage, at $4.2 \mathrm{~K}$ and $15 \mathrm{~K}$ respectively, insets show $\mathrm{R}(\mathrm{T})$ behaviour at $0 \mathrm{~V}$; (e) position of peaks vs temperature in both large FIB and RM samples of (d).

transport in larger RM and FIB constrictions, of lateral dimensions $1 \mu \mathrm{m} * 0.5 \mu \mathrm{m}$ and $8 \mu \mathrm{m} * 1 \mu \mathrm{m}$ respectively, whose zero-bias resistance is closer to the expected bulk behaviour. Nonetheless, the observed transport through each of these constrictions clearly shows peaks at around the same voltages as observed in the insulating constrictions, which also have similar temperature dependences (see Fig. 1(e)) despite their higher absolute conductance.

We conclude that the size solely determines the behaviour of electrical transport in these systems, independent of constriction-fabrication method. At small sizes constrictions behave like CDW-insulatorCDW tunnel barriers, showing expected $\mathrm{dI} / \mathrm{dV}$ peaks at twice the CDW energy gaps. As constriction size increases, low bias transport crosses over to bulk-like behaviour and the system forms a CDW-normal metal and normal metal-CDW junction in series, again contributing $\mathrm{dI} / \mathrm{dV}$ features at twice the CDW energy gaps.

\section{Acknowledgments}

We thank S. V. Zaitsev-Zotov and S. Artemenko for fruitful discussions. This work was supported by INTAS-NIS, the Netherlands Organization for Scientific Research (NWO) and the National Science Foundation (NSF). We thank J. van Ruitenbeek for the use of the equipment in the MCBJ work.

\section{References}

[1] A. A. Sinchenko et al., Phys. Rev. B 60, 4624 (1999).

[2] S. V. Zaitsev-Zotov et al., JETP letters 73, 25 (2001).

[3] J. P. Sorbier et al., Phys. Rev. Lett. 76, 676 (1996).

[4] K. O'Neill et al., J. Phys. IV (France) 12, 185 (2002); J. M. Ruitenbeek et al., Rev. Sci. Instrum. 67, 108 (1996). 